\title{
As incitações das circunspecções na atenção primária no sistema único de saúde
}

\author{
The incitations of circumspections in primary care in the unique health system
}

Las incitaciones de las circunstancias en la atención primaria en el sistema de salud único

José Milton de Aquino e Silva Neto ${ }^{1 *}$, Amanda da Silva Costa ${ }^{1}$, Samila dos Santos Araújo ${ }^{1}$, Alfredo Jose Pereira Filho ${ }^{1}$, Michelle Leão Bittencourt Brandão Medeiros ${ }^{1}$, Tayguara Cerqueira Cavalcanti ${ }^{1}$.

\section{RESUMO}

Objetivo: Examinar e revisar as colaborações da Estratégia de Saúde da Família e retratar fatores dos quais conseguem sensibilizar o desenvolvimento sustentável do modelo em questão. Métodos: Relacionou-se à uma revisão de literatura, com indagações explicativas e informativas, dos quais os processos de caracterizações foram definidos por meio de buscas por literaturas cientificas, em bases de dados, tendo como descritores: Assistência ao Paciente, Atenção Primária à Saúde, Sistema Único de Saúde. Resultados: A princípio foi analisado a forma de grandeza, dos quais foram destacadas as colaborações para a amplificação das atenções primárias, regularização das conjecturas estabelecidas e o cuidado na equidade e incitações referentes a forma de contribuição, didática, gestão pessoal e à organização multiprofissional. Analisou-se aumento de serviços, atenções de ações programadas e auxílio integrativa da atenção na esfera organizacional, possuindo como desafios referente a porta de entrada, inclusão aos serviços e participações sociais. Considerações finais: No assistencialismo foi considerado as vantagens do acolhimento multidisciplinar, receptividade, conexão, forma humanitária, direcionamento comunitário. Os obstáculos para seu aperfeiçoamento se condicionam a fatores interligados entre paciente e profissional exigindo maior atenção e esforço das políticas do Estado.

Palavras-chave: Assistência ao paciente, Atenção primária à saúde, Sistema único de saúde.

\begin{abstract}
Objective: Examine and review the collaborations of the Family Health Strategy and portray factors of which they are able to raise awareness of the sustainable development of the model in question. Methods: Related to a literature review, with explanatory and informative questions, of which the characterization processes were defined through searches for scientific literature, in databases, having as descriptors: Patient Care, Primary Health Care, Unified Health System. Results: At first, the form of greatness was analyzed, of which the collaborations for the amplification of the primary attention, regularization of the established conjectures and the care in equity and incitations regarding the form of contribution, didactics, personal management and the Multiprofessional organization were highlighted. An increase in services, attention to programmed actions and integrative assistance in the organizational sphere were analyzed, with challenges related to the entrance door, inclusion in services and social participation. Final considerations: In assistance, the advantages of multidisciplinary reception, receptivity, connection, humanitarian form, community orientation were considered. The obstacles to its improvement are conditioned by factors interconnected between patient and professional, requiring greater attention and effort from State policies.
\end{abstract}

Key Words: Patient care, Primary health care, Unified health system.

${ }^{1}$ Centro Universitário CESMAC (CESMAC), Maceió - AL. *E-mail: milton_neto_166@hotmail.com 


\section{RESUMEN}

Objetivo: Examine y revise las colaboraciones de la Estrategia de salud familiar y describa los factores que logran para crear conciencia sobre el desarrollo sostenible del modelo en cuestión. Métodos: Relacionado con una revisión, con preguntas explicativas e informativas, de los cuales los procesos de caracterización se definieron a través de búsquedas de literatura científica, en bases de datos, que tienen como descriptores: Atención al Paciente, Atención Primaria de Salud, Sistema Único de Salud. Resultados: Al principio, se analizó la forma de la grandeza, de las cuales se destacaron las colaboraciones para la amplificación de la atención primaria, la regularización de las conjeturas establecidas y el cuidado en la equidad e incitaciones con respecto a la forma de contribución, didáctica, gestión personal y la organización multiprofesional. Se analizó un aumento en los servicios, atención a acciones programadas y asistencia integradora en el ámbito organizacional, con desafíos relacionados con el portal, inclusión en servicios y participación social. Consideraciones finales: En asistencia, se consideraron las ventajas de la recepción multidisciplinaria, receptividad, conexión, forma humanitaria, orientación comunitaria. Los obstáculos para su mejora están condicionados por factores interconectados entre el paciente y el profesional, que requieren una mayor atención y esfuerzo de las políticas estatales.

Palabras clave: Atención al paciente, Atención primaria de salud, Sistema único de salud.

\section{INTRODUÇÃO}

Em 1978, o lançamento da "Declaração de Alma Ata" provocou uma demarcação bastante crucial no progresso da atenção primária à saúde (APS) em todos os continentes, essa declaração visou defender a APS como uma forma de elemento central dos quais se referia ao sistema de saúde. A alegação propôs a idealização centralizada de melhorias no sistema de saúde contemporâneo e contribuindo assim para atingir e estabelecer repercussão positiva de saúde mais benéficas para o usuário e tornou-se ela mais igualitária, tornando melhor a eficiência, a eficácia e a contentamento dos usuários (CONILL EM, 2008).

Com o objetivo principal de contribuição desses programas e melhorar a sua forma de capacitação referente as deliberativas do foco principal que seria a ajuda para todos, foram estabelecidos programas de assistencialismo no país, sendo eles os Núcleos de Apoio à Saúde da Família (NASF), que possui como um dos propósitos favorecer o assistencialismo nas equipes multidisciplinares de saúde da família na concretização de estabelecimentos de redes de serviços e amplificar o domínio e a finalidade das ações da Atenção Primária à Saúde (APS) (ARANTES LJ, et al., 2016).

No entanto, é importante destacar a existência de diferentes métodos para alcançar a APS. Em alguns países/regiões, a atenção primária à saúde é considerada um programa seletivo do qual é focado no processo de assistencialismo dos serviços para as pessoas mais carentes, em outros casos, é o primeiro nível do sistema de saúde em que presta serviços clínicos sendo responsáveis por coordenar os cuidados cabíveisde atenção em seu processo de organização e estratégias naspolíticas de administração das modalidades assistenciais (BRASIL, 2017b).

O ponto de vista atual relacionado ao processo de desenvolvimento da atenção primária à saúde (APS) foi proposto pela primeira vez na descrição de Dawson, em (1920), no Reino Unido, sendo sugerido uma classificação do sistema de saúde mais ampla (DEMARZO MMP, 2011). Foi dado ênfase em muitos pontoschave dos cuidados de saúde ao usuário, incluindo: atendimento domiciliar, pontos de atendimento primário de saúde, centros de atendimento secundário, o atendimento prestado nesse último nível é considerado um dos mais complexo, com isso os pacientes têm contato direto com profissionais de saúde, sendo seguidos padronizações de serviços complementares padronizados, como eficácia, eficiência, acessibilidade e segurança do paciente (MENDES EV, 2015).

Devido ao seu potencial, o Programa Saúde da Família (PSF) é afamado como sendo a Estratégia de Saúde da Família (ESF), pois consegue direcionar o sistema de organização de saúde, proporcionando soluções para todas as indispensabilidades de saúde da sociedade e auxilia a modificar o atual modelo de atenção. Para tanto, a ESF assenta-se nas concepções norteadoras do progresso de práticas em saúde, 
como o seu foco principal não só do paciente de forma particularizada, mas também em sua família, o contato com o usuário, a forma de completude e administração da atenção, a inter-relação com a rede de assistencialismo, cooperação social e desempenho intersetorial (GIOVANELA L, et al., 2009).

É necessário considerar que, após 20 anos de implementação, a estratégia foi defendida como o principal conteúdo da agenda política para a organização dos serviços e ações de atenção primária à saúde no Brasil e produziu vários resultados benéficos para a saúde das pessoas. No entanto, por estar inserida em cenários complexos e diversos e apresentar muitos benefícios de interesses políticos sociais e econômicos, ocasionalmente algumas de suas competências conseguem serem diminuídas, o que tem levado a várias indagações quanto à sua forma de confiabilidade como organização construtiva dos assistencialismos e de suas condutas de saúde, sendo sucessor do padrão habitual de APS no país.

Perante essa forma de entendimento, torna-se indispensável analisar a ESF, constatando as áreas susceptíveis de interferência das competências sanitárias com intuito ao seu póstero aprimoramento (ALMEIDA PF, 2017).

Portanto, este artigo tem como objetivo distinguir e examinar as imprescindíveis colaborações e instigações da Estratégia de Saúde da Família para amelhor evolução da atenção primária à saúde no Brasil, com a finalidade de retratar sobre diversos fatos dos quais conseguem sensibilizar o desenvolvimento sustentável do modelo em questão.

\section{MÉTODOS}

Refere-se a uma busca bibliográfica determinada por meio de uma revisão de literatura com abordagem descritiva e caráter informativo efetivada entre os intervalos de fevereiro de 2020 a julho de 2020, dos quais analisou-se artigos publicados em bases de dados eletrônicos, Literatura Latino Americana e do Caribe em Ciências da Saúde (LILACS), Literatura Internacional em Ciência da Saúde (MEDLINE) e Scientific Electronic Library Online (SCIELO) e livros que estavam disponíveis nos acervos bibliográficos presentes na biblioteca central do Centro Universitário CESMAC (Centro de Ensino Superior de Maceió).

Para tal fim, o princípio do estudo foi estabelecido através dos seguintes métodos: foi levado em consideração teses, monografias, emissões de publicações do Ministério da Saúde e Organizações Internacionais, no idioma inglês, espanhol e português, optando-se pelos trabalhos que foram publicados nos últimos 10 anos (2010 a 2020). Em concordância com a sua formulação organizacional, metodológica e descrita por Silva Neto JM, et al., (2019), o estabelecimento dos artigos foram provenientes através de 5 pesquisadores: a princípio foi determinado o tema a ser observado, examinado e consecutivamente, foram realizadas as leituras devidas de forma profunda concernente com os resumos para o aproveitamento dos processos descritivos, tornando-os assim mais restritos.

Logo em seguida foram estabelecidas as leituras de todos os artigos englobados, como também foram verificadas as listas de referências, com o intuito de analisar artigos que referia a presente revisão. Com os artigos definidos, foram compostos as partes das centrais variáveis de solicitações, como a extensão das amostragens, o objetivo, a metodologia, as normas esclarecidas e os fundamentais resultados, que se exibiram a Atenção Primária do Sistema Único de Saúde (SUS), tendo como métodos de restrições os períodos de publicações, ensaios não controlados e os artigos que não possuíam relevância com a temática, nos artigos aprovados foram observados as políticas de humanização, tratamento ao paciente, funcionamento do SUS. Os descritores utilizados foram: Assistência ao Paciente, Atenção Primária à Saúde, Sistema Único de Saúde.

\section{RESULTADOS E DISCUSSÃO}

Para o progresso dessa mencionada revisão de literatura, estabeleceu-se 30 artigos científicos que assegurava a veracidade e completude nas bases de dados referidas. Ao transcorrer do processo de evolução da pesquisa, 14 dispunham duplicidade nas referidas fontes de embasamento para à pesquisa, dispondo assim de 16 artigos para o processo estimado do respectivo material, contudo 6 foram eliminados por não dispor de forma clara com as diretrizes indispensáveis da composição do estudo. 
Em decorrência 10 artigos foram verificados para a avaliação através de sínteses que estavam presentes, desses, um foi excluído, deste modo, definiu-se 9 artigos para o esclarecimento e constituição indispensável para o aperfeiçoamento do estudo. Logo em seguida, após a organização das circunstâncias do respectivo estudo, não foram aplicadas outras fontes de pesquisa ao perceberas verificações informativas que se apresentaram a devida pesquisa, a revisão foi ajustada por 6 artigos científicos de conceituada importância, e o fluxograma da Figura 1 assevera as formas de pesquisas que foram geradas de acordo com o apuramento efetivado por explorações estabelecidas para a legitimação direta deste estudo literário.

Figura 1 - Fluxograma dos estudos identificados.

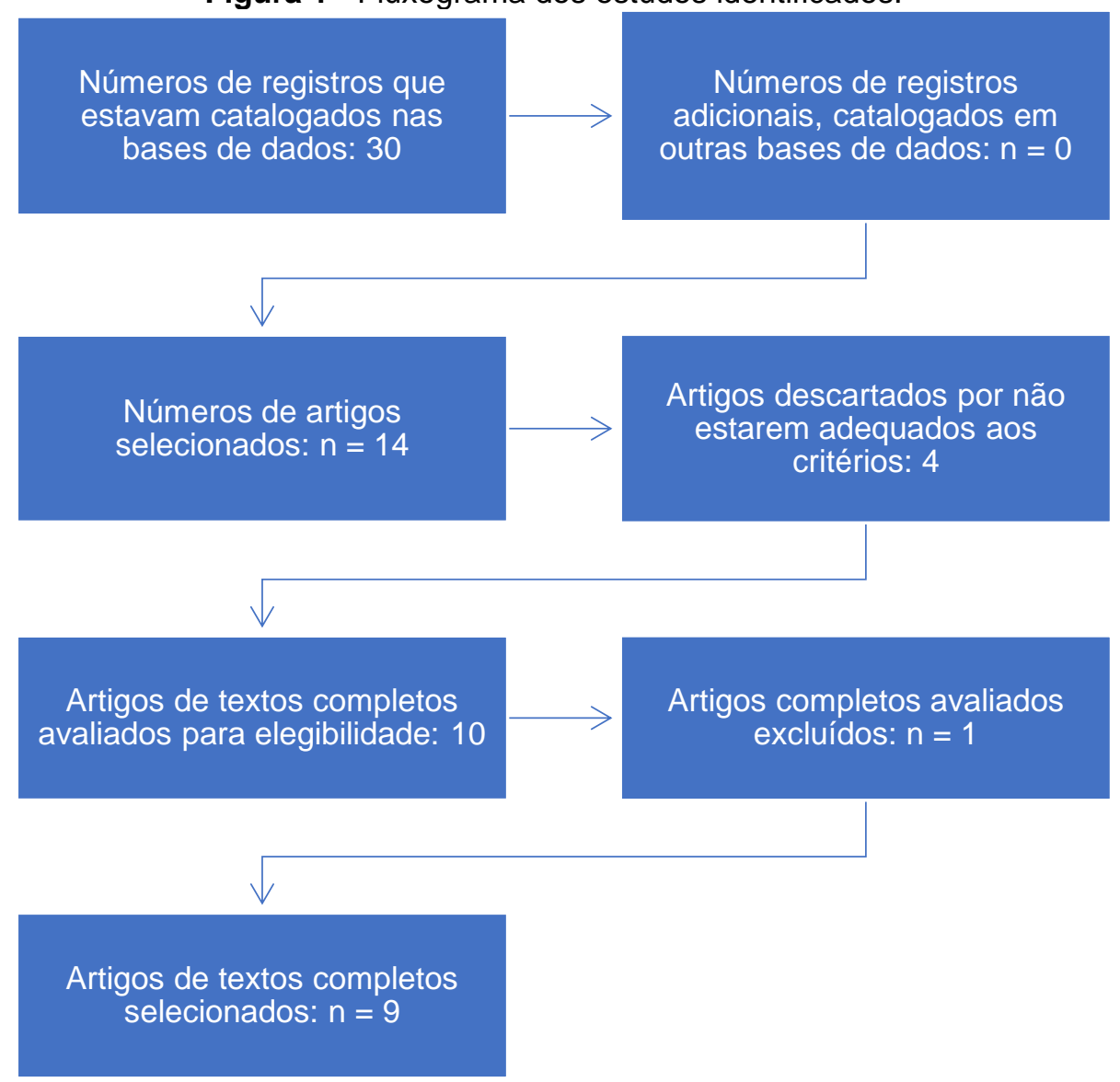

Fonte: Neto JMAS, et al., 2020.

Os artigos possuíram suas informações através dos tópicos: artigo/autor, ano, periódico, objetivo e resultados, os quais foram tabulados e apresentados na Tabela 1. 
Tabela 1 - Delineamento, métodos e principais desfechos dos estudos selecionados.

\begin{tabular}{|c|c|c|c|c|}
\hline Artigo/Autor & Ano & Periódico & Objetivo & Resultados \\
\hline $\begin{array}{l}\text { Educação, saúde e ambiente: as } \\
\text { concepções dos agentes comunitários } \\
\text { de saúde (GUIMARÃES FT, et al.). }\end{array}$ & 2013 & $\begin{array}{l}\text { Ensino, saúde } \\
\text { e ambiente }\end{array}$ & $\begin{array}{l}\text { Analisar as concepções dos agentes } \\
\text { comunitários de saúde (ACS), de quatro } \\
\text { unidades da Estratégia de Saúde da } \\
\text { Família (ESF) do município de } \\
\text { Teresópolis-RJ, acerca da relação entre } \\
\text { o ambiente e o processo saúde-doença. }\end{array}$ & $\begin{array}{l}\text { Pôde-se observar que as percepções dos ACS são } \\
\text { consistentes, no sentido de correlacionar a existência de um } \\
\text { ambiente degradado com o processo saúde-doença. }\end{array}$ \\
\hline $\begin{array}{l}\text { A importância do acolhimento aos } \\
\text { usuários da atenção básica: uma } \\
\text { assistência humanizada (BRAZ FAF). }\end{array}$ & 2013 & $\begin{array}{l}\text { Universidade } \\
\text { Federal de } \\
\text { Minas Gerais }\end{array}$ & $\begin{array}{l}\text { Analisar à importância do acolhimento } \\
\text { aos usuários da atenção básica: uma } \\
\text { assistência humanizada. }\end{array}$ & $\begin{array}{c}\text { A importância do acolhimento aos usuários da atenção básica: } \\
\text { uma assistência humanizada. }\end{array}$ \\
\hline $\begin{array}{l}\text { A posição da Estratégia Saúde da } \\
\text { Família na rede de atenção à saúde na } \\
\text { perspectiva das equipes e usuários } \\
\text { participantes do PMAQ-AB (FAUSTO } \\
\text { MCR, et al.). }\end{array}$ & 2014 & Saúde debate & $\begin{array}{c}\text { Analisa a posição da Estratégia Saúde } \\
\text { da Família na rede de atenção à saúde } \\
\text { sob a perspectiva das } 16.566 \text { equipes de } \\
\text { Saúde da Família e dos } 62.505 \text { usuários } \\
\text { participantes do Programa Nacional para } \\
\text { Melhoria do Acesso e da Qualidade da } \\
\text { Atenção Básica em } 2012 \text {. }\end{array}$ & $\begin{array}{l}\text { Indicam que as equipes atuam cada vez mais como porta de } \\
\text { entrada preferencial, atendendo a demandas diversas e } \\
\text { exercendo a função de filtro para a atenção especializada. }\end{array}$ \\
\hline $\begin{array}{c}\text { Análise da eficiência na atenção } \\
\text { primária à saúde sob a ótica dos } \\
\text { profissionais da área (CABRAL KFD, et } \\
\text { al.). }\end{array}$ & 2016 & $\begin{array}{l}\text { Revista de } \\
\text { Gestão em } \\
\text { Sistemas de } \\
\quad \text { Saúde }\end{array}$ & $\begin{array}{l}\text { Analisar a eficiência da Atenção Primária } \\
\text { à Saúde, avaliando as experiências de } \\
\text { municípios brasileiros do estado de } \\
\text { Minas Gerais no que se refere à sua } \\
\text { eficiência na promoção da saúde. }\end{array}$ & $\begin{array}{l}\text { Observou-se que as dificuldades apontadas em comum nos } \\
\text { municípios analisados se referem à falta de recursos ou sua } \\
\text { limitação, bem como às condições educacionais da população } \\
\text { e sua baixa participação e conhecimento sobre a Estratégia } \\
\text { Saúde da Família. Conclui-se que há grande necessidade de } \\
\text { trabalhos intersetoriais que apoiem ações de promoção da } \\
\text { saúde já existentes, bem como novas estratégias que podem } \\
\text { elevar o nível de conhecimento da população, dos } \\
\text { profissionais e também dos gestores como forma de se } \\
\text { melhorar o desempenho da promoção da saúde. }\end{array}$ \\
\hline $\begin{array}{c}\text { A qualidade da atenção primária no } \\
\text { programa mais médicos- a experiência } \\
\text { dos médicos e usuários } \\
\text { (ORGANIZAÇÃO PAN-AMERICANA } \\
\text { DA SAÚDE). }\end{array}$ & 2017 & $\begin{array}{l}\text { Biblioteca } \\
\text { Virtual da } \\
\text { Saúde (BVS) }\end{array}$ & $\begin{array}{c}\text { Avaliar a força da APS à saúde nas } \\
\text { equipes das estratégias saúde da família } \\
\text { de todo Brasil, por meio da experiência } \\
\text { de usuários dos serviços e de médicos } \\
\text { cubanos participantes do programa mais } \\
\text { médicos. }\end{array}$ & $\begin{array}{l}\text { As estratégias nacionais para ampliação e qualificação da } \\
\text { APS no Brasil têm feito escores mais altos na maioria das } \\
\text { regiões, apesar da dificuldade de acesso às Unidades de } \\
\text { Saúde da Família, aqueles pacientes que conseguem acessá- } \\
\text { las desenvolvem relações de confiança com os médicos, } \\
\text { como demonstram os Escores de Longitudinalidade. }\end{array}$ \\
\hline
\end{tabular}

REAS/EJCH | Vol.12(10) | e4679 | DOI: https://doi.org/10.25248/reas.e4679.2020 Página 5 de 10 
Revista Eletrônica Acervo Saúde / Electronic Journal Collection Health | ISSN 2178-2091

\begin{tabular}{|c|c|c|c|c|}
\hline Artigo/Autor & Ano & Periódico & Objetivo & Resultados \\
\hline $\begin{array}{l}\text { Estratégias para consolidação da } \\
\text { coordenação do cuidado pela atenção } \\
\text { básica (ALMEIDA PF, et al.). }\end{array}$ & 2017 & $\begin{array}{l}\text { Scientific } \\
\text { Electronic } \\
\text { Library } \\
\text { (SCIELO) }\end{array}$ & $\begin{array}{l}\text { Avaliar a organização da porta de } \\
\text { entrada, resolutividade e continuidade do } \\
\text { cuidado, integração horizontal, } \\
\text { organização dos fluxos e acesso à rede } \\
\text { de referência, continuidade informacional } \\
\text { e comunicação entre profissionais }\end{array}$ & $\begin{array}{l}\text { Os resultados indicaram que a atenção primária em saúde se } \\
\text { consolidou como porta de entrada preferencial. }\end{array}$ \\
\hline $\begin{array}{l}\text { Avaliação da atenção primária: o ponto } \\
\text { de vista de usuários (GONTIJO TL, et } \\
\text { al.). }\end{array}$ & 2017 & Saúde Debate & $\begin{array}{c}\text { Avaliar atributos da Atenção Primária à } \\
\text { Saúde (APS), sob a percepção de } \\
\text { usuários. }\end{array}$ & $\begin{array}{l}\text { Conclui-se que os atributos da APS analisados receberam } \\
\text { avaliação heterogênea e a maioria necessita de um olhar mais } \\
\text { atento e investimento por parte dos gestores e profissionais. }\end{array}$ \\
\hline $\begin{array}{c}\text { Percepções de usuários atendidos em } \\
\text { um Pronto Atendimento: olhar sobre a } \\
\text { Atenção Primária à Saúde (BARATIERI } \\
\text { T, et al.). }\end{array}$ & 2017 & $\begin{array}{l}\text { Revista de } \\
\text { Saúde } \\
\text { Pública do } \\
\text { Paraná }\end{array}$ & $\begin{array}{l}\text { Compreender os motivos que levam os } \\
\text { usuários a buscar as Unidades de Pronto } \\
\text { Atendimento (UPA) quando o problema } \\
\text { de saúde poderia ser resolvido no âmbito } \\
\text { da Atenção Primária à Saúde (APS). }\end{array}$ & $\begin{array}{l}\text { Descompasso entre a visão médico-centrada e a inadequação } \\
\text { das equipes de saúde da APS para atenção à demanda } \\
\text { espontânea; acolhimento deficitário na APS gera dificuldades } \\
\text { de acesso e baixa resolutividade e desarticulação entre UPA e } \\
\text { APS. Necessidade de estruturação da coordenação do } \\
\text { cuidado. Conclui-se que as UPAs são vistas pela população } \\
\text { como mais resolutivas, havendo necessidade de ordenar } \\
\text { fluxos de atendimento e educar/informar a população sobre } \\
\text { seu funcionamento. }\end{array}$ \\
\hline $\begin{array}{l}\text { Atenção Primária à Saúde na Agenda } \\
\text { Pública Brasileira: dilemas entre } \\
\text { focalização e universalidade (SENNA } \\
\text { CMM e SANTOS AM). }\end{array}$ & 2017 & $\begin{array}{l}\text { Revista de } \\
\text { Políticas } \\
\text { Públicas }\end{array}$ & $\begin{array}{l}\text { Reflexão sobre os desafios postos à } \\
\text { Atenção Primária à Saúde no contexto } \\
\text { brasileiro. }\end{array}$ & $\begin{array}{l}\text { Houve um esforço importante de expansão da oferta de } \\
\text { serviços de saúde da APS nas últimas décadas, mas, } \\
\text { observa-se que essa expansão se deu de forma desigual e } \\
\text { heterogênea no território nacional. As recentes iniciativas de } \\
\text { constituição das Redes de Atenção à Saúde confirmam o } \\
\text { ainda frágil desempenho da APS como eixo organizador da } \\
\text { rede de serviços de saúde e política universal. Tal } \\
\text { reconhecimento exige recuperar uma perspectiva crítica de } \\
\text { análise sobre os constrangimentos que se impõem ao SUS. }\end{array}$ \\
\hline
\end{tabular}

Fonte: Neto JMAS, et al., 2020.

REAS/EJCH | Vol.12(10) | e4679 | DOI: https://doi.org/10.25248/reas.e4679.2020 Página 6 de 10 
O Sistema Único de Saúde (SUS) possuiu como foco principal em seu desenvolvimento servir de exemplo para ao que se referia a nova forma de desenvolvimento e inovação dos quais determinaria a nova forma de ordenação social, porém foi bastante impactado com o contexto histórico e as questões culturais referentes a forma de vida que a população em massa deparava-se, em que a desigualdade, a falta de humanidade e a descriminação eram seccionadas ao que se condiz com a atenção básica da saúde.

Com isso as introduções na constituição do assistencialismo por completo relacionado ao Sistema Único de Saúde, desde o início foi apresentado como sendo aprincipal forma de estabelecer a dignidade da população, possuindo como forma crucial a vontade demodificar as padronizações existentes do hospitalocêntrico dos quais só tomava providencias quando o paciente apresentava sinais ou sintomas de determinadas doenças e os que não possuíam condições econômicas, muito das vezes não tinha nem abertura para cuidados paliativos, chegando a um elevado índice de óbitos (MENICUCCI TMG, 2009).

Nos dias atuais as formas de condutas prestadas ao SUS são estabelecidas através de uma ótica e olhar crítico dos quais são englobados o envolvimento de equipes multidisciplinares na atenção básica. A rede de cuidados à saúde é formada através de disponibilizações de prestações de serviços em cada macro e microrregiões, dentre eles: a consulta de forma domiciliar, unidade de assistencialismo básico, centro de especializações multiprofissionais e os hospitais. Essa padronização serve como forma de amparo para a comunidade, visto que a maior parcela da população é atendida por esses centros, principalmente os usuários que dispõem de poder socioeconômico abaixo da média nacional, sendo à atenção primária à saúde a porta de entrada para os cuidados necessários e integrativos para a população (BRASIL, 2011a).

Diante desse aspecto observa-se que um dos momentos mais marcantes no conceito histórico que circundou a APS decorreu, no entanto, ao final da década de 70 , onde sucedeu a primeira reunião de conferencia a nível Internacional em que compreendia contextos temáticos referentes as dimensões que necessitariam serem ocupadas ao atendimento das atenções primárias que necessitariam estarem implantadas, do qual, até o respectivo instante era apenas delineada como 'Alma-Ata', esse título foi estabelecido pelo motivo da conferencia ser sediada na cidade onde localiza-se no Cazaquistão (FAUSTO MCR, et al., 2014).

Nesse cenário renovador, 134 países envolveu-se, buscando o bem-estar das populações e fixaram um documento do qual se estabelecia a diretriz de melhorar as formas de assistencialismo referente aos programas de saúde, buscando ser estabelecida com clareza e objetividade, dos quais consideraram uma agregação de valores e concepções que amplificavam o verdadeiro interpretação e conceitualização relacionado à saúde, adaptando-se as particularidades socioeconômicas de cada país (ALMEIDA PF, et al., 2017).

De acordo com o trabalho seminal, os cuidados e deveres relacionados a saúde estabelecida de forma primária devem dispor de prestação de serviços à cada comunidade de forma particularizada, visando as peculiaridades de cada uma, observa-se que hoje existem centros de atendimento primário tanto nos espaços rurais quanto no meio urbano.

A atenção primária à saúde tem como objetivo dispor de prestação de atenção nos serviços comunitários que abrange a saúde em um contexto geral, desde o desempenho de cuidados pré-natais, consulta preventiva e educacionais, acompanhamento de possíveis epidemias. Consequentemente, é de valia destacar que, mesmo depois de muito tempo após sua implantação, a APS continua a ter ainda hoje, grandes desafios para a saúde pública na maioria dos países país (SENNA CMM e SANTOS AM, 2017).

\section{A importância da a Estratégia Saúde da Família e suas inter-relações com Programa de Agentes Comunitários de Saúde}

O Brasil vem desempenhando um papel bastante relevante, visando sempre melhorias da saúde pública em nível da APS, focalizando na prevenção, precauções e assistencialismo, destacando-se as inter-relações do trabalho do Programa Agentes Comunitários de Saúde (PACS), a Estratégia Saúde da Família (ESF) e o Núcleo de Apoio à Saúde da Família (NASF). O Programa Agentes Comunitários de Saúde é um padrão dos quais vem sendo contínuo pelo Ministério da Saúde, onde é operante em diversos graus da esfera nacional. 
Além dos Estados os municípios de cada região vêm adquirindo repercussões positivas e benéficas fazendo com quer esse programa seja transformado em um dos empenhos mais eficazes de saúde.

Desde a implementação do PACS, a taxa de mortalidade infantil foi diminuída, o nível de imunização aumentou e a velocidade da intervenção ocupacional acelerou, aprimorando diretamente a APS (BARATIERI T, et al., 2017).

O trabalho da ACS não é algo novo e muito menos limita-se apenas no Brasil, esse trabalho possui inúmeras nomenclaturas em diferentes países, porém possui basicamente as mesmas especificidades e funcionalidade, sendo reconhecido essa função à nível internacional, sendo reconhecido pela equipe de pesquisa da Organização Mundial da Saúde (OMS), em que estabelece a ACS como sendo uma atividade responsável de necessária em todos os locais, dos quais analisa a saúde dos indivíduos de cada comunidade distinta (SENNA CMM e SANTOS AM, 2017).

No Brasil, o Programa de Agentes Comunitários de Saúde foi adotado no início da década de 90, através do estabelecimento de convênio entre a Fundação Nacional de Saúde e as Secretarias de Estado da Saúde, dos quais possuí uma equipe multidisciplinar cuja a sua organização era estabelecida por 1 enfermeiro que coordenava 30 ACS, em uma Unidade Básica de Saúde. O início de sua implantação foi ocorrido inicialmente no Norte do país, isso aconteceu devido à grande epidemia de cólera que estava ocorrendo na época, sendo observado que as áreas que estavam sendo acompanhadas por essa equipe obteve resultados bastantes significativos e positivos, em relação aos que não tiveram acompanhamento da equipe (MARTINES WRV e CHAVES EC, 2007).

Entre as formas de consentimentos básicos dos ACS's na ajuda a atenção primária à saúde, considerase as funcionalidades de esquematizar sua área de execução, registrar e catalogar os usuários de determinada família, identificando pacientes com doenças sistêmicas, pacientes de riscos, conduzir aquela população sobre a utilização de forma propicia dos serviços de saúde ofertados, conduzir um acompanhamento continuo dos usuários em sua seriedade e comunicar os demais componentes da equipe multidisciplinar sobre as circunstâncias destes, verificar atuações e condutas sobre a educação à saúde; proporcionar palestras comunitárias, O ACS deve possuir a capacidade de identificar riscos potenciais em suas microáreas sendo ela de forma física ou social para só assim o sistema básico de saúde ficar atento a realidade da sua localidade (GUIMARÃES FT, et al., 2013).

\section{A importância da atenção primária à saúde}

No entanto, atenta-se que a APS nos dias atuais é um dos fundamentais aspectos de acesso dos usuários ao SUS, existindo desta maneira um lugar dos quais pode-se centralizar processos de conexões para a melhora na assistência. Com isso, o sistema de atenção primária visa de forma direta nortear e acompanhar os seus usuários relacionando-se e respeitando as atitudes que circundam a conceitualização do ingresso e buscando formas ordenadas para poder impulsionar a atenção ao paciente, visando estabelecer encadeamento, continuidade e integralidade dos usuários (ALMEIDA PF e MARIN J, 2017).

Desejando determinar esse conjunto de valores, a ABS fazem implementações de programas, execução e conjunto de condutas, tendo em consideração em todo o tempo as questões sociais, culturais e econômicos, buscando desta forma sempre a imparcialidade nas diferentes formas de carências (seja ela qual for) de cada indivíduo que busca o Sistema de saúde (SENNA CMM e SANTOS AM, 2017).

A efetividade da APS possui seguimentos benéficos no respectivo grau de atuação, da mesma maneira que decorrências positivas sobre níveis mais elevados, como é o caso da atenção as atividades desempenhadas de média e alta complexidade (OPAS, 2018).

O funcionamento da atenção primária à saúdeé refletida nas internações por situações fragilizadas a esse grau de precaução na hora da atenção,desse modo, a competência da APS pode ser capaz de diminuir o avanço de determinada patologia e por consequência reduz as internações no âmbito hospitalar, como também o uso de intervenções medicamentosas e terapêuticas mais dispendiosos, aumentando assim os gastosno sistema de saúde (FAUSTO MCR, et al., 2014). 
Os estudos estabelecidos por Rosa WDAG e Labate RC (2005), demostraram que a atenção primária à saúde alcança cerca de $80 \%$ a $85 \%$ na solução das crises na adversidade de saúde da população assistida. No entanto, esses autores salientam que, para que ocorra esse efeito benéfico a grau de solução, a APS necessita está bastante eficaz.

As questões que compreendem os processos de tangibilidade dos acontecimentos que contornam às organizações governamentais pertencentes a saúde fazendo com que seja acertada com a reiteração no alto esclarecimento, por procedimentos de exemplificações no sistema público de saúde no que diz respeito ao elevado padrão liberal dos quais contém aspectos filosóficos políticos e sua forma moral fundamentada na independência, com a aprovação das comissões responsáveis do Estado e procurando em todo o tempo a conformidade perante da lei, dessa maneira percebe-se que apenas os enfrentamento contínuos das ações comunitárias pelo direito à saúde conseguem atestar uma superior assistência e acompanhamento (BRAZ $\mathrm{FAF}, 2013)$.

\section{Obstáculos encontrados na APS}

Pode ser analisado o respectivo cotidiano dos colaboradores e trabalhadores da área da saúde é necessário observar a forma de atuação continua e constante desses profissionais, sendo à APS a principal forma de porta de entrada no primeiro contato em uma determinada consulta, dos quais pode-se observar que existem inúmeras procuras e a equipe multiprofissional desempenha a forma de funcionamento por meio de processos de filtragem dos pacientes que mais precisam por conseguinte, estabelecer o seguimento do encaminhamento ao local de atendimento especializado (DIAS OV, et al., 2016).

Ainda assim, encontram-se significativos impedimentos organizacionais para a efetivação das específicas assistências na hora do atendimento, um dos enfoques relacionados a essas causas podem encontrarem-se na ausência de ordenações de fluência, a integração abrangedores da APS às redes mais especialistas em que mantêm-se sendo introdutórios e encontra-se uma carência de interligação entre à Atenção Primária à Saúde e as equipes especializadas (FAUSTO MCR, et al., 2014).

\section{Acolhimento ao paciente}

Com o propósito de observar as características da intensificação da APS na estratégias de saúde da família no Brasil, por intermédio de organizações, estruturas e técnicas determinadas em padronizações nacionais para ampliar a ponderação da APS, demonstrando assim confirmações que no Brasil está possibilitando formas incrementais possuindo capacidade e avaliações mais respeitadas na maioria das regiões, ainda que encontre-se adversidades nos pacientes na entrada das Unidades de Saúde da Família, porém é eminente que osusuários que obtêm o estabelecimento da verdadeira prestação de serviços adquirem uma correlação de confiabilidade com toda equipe de saúde envolvida (OPAS, 2018).

Na visão determinada por Braz FAF (2013) o atendimento e a forma de acolhimento, pode ser amplificado na hora do primeiro contato entre os usuários do Sistema Único de Saúde na atenção primária, dos quais pode-se ser associado e observado de acordo com a regularidade, frequência no acompanhamento dos pacientes, com isso é importante que os profissionais de saúde estejam qualificados não só na hora do atendimento em si, como também observar a forma que o paciente se posiciona, tentando ouvir de forma ativa o que levou a ele a ir buscar tão consulta, para só assim entender e definir à suabusca, respeitando sempre as diretrizes que o processo de atendimento humanizado necessita, demonstrando o amparo que 0 local e o profissional oferece.

O regulamento dos quais decorrem os processos avaliativos concernentes ao contentamento do paciente/usuário é detalhado e esquematizado, dos quais apresentam como responsabilidades de transcorrer por meio de propostas de assegurar para a obtenção doresultado final. Um dos instrumentos que são competentes para essa mensuração de contentamento demandam estarem assentados na compreensão do paciente quando expõem suavisão a respeito da natureza das ocupações ao qual foi submetido no respectivo atendimento. As expectativas dos usuários são feitas de forma individualizada pelo meio da contextualização do engajamento do profissional, dos conhecimentos adquiridos e vivenciados nos atendimentos anteriores e do nível da instrução do paciente (BRUNHAUSER AL, et al., 2013). 


\section{CONSIDERAÇÕES FINAIS}

Verificou-se que, no Brasil, a atenção primária à saúde é uma das principais portas de entrada dos usuários sendo um dos serviços fundamentais na prestação de atendimentos aos usuários que mais precisam, visto que o assistencialismo é considerado fundamental evitando assim possíveis diagnósticos tardios, existem inúmeras vantagens nessa forma de abordagem e entre elas são destacados o acolhimento multidisciplinar, multiprofissional, a forma de receptividade, conexão, forma humanitária de atendimento, direcionamento comunitário, porém existem obstáculos para seu aperfeiçoamento dos quais se condiciona a fatores interligados entre paciente e profissional exigindo maior atenção e esforço das políticas do Estado, Ficando desta forma evidente que deve-se estabelecer um debate mais sólido em relação aos distintos aspectos ao que se refere a acessibilidade do paciente da APS e do mesmo modo enxergar condutas de aperfeiçoamentos tanto ao que se refere a forma de planejar quanto nas correlações entre a equipe e os usuários que procuramsuporte e atendimento no SUS.

\section{REFERÊNCIAS}

1. ALMEIDA PF, MARIN J. Estratégias para Consolidação da Coordenação do Cuidado pela Atenção Básica. Trabalho. Educação. Saúde. 2017; 15(2): 373-398.

2. ARANTES LJ, et al. Contribuições e desafios da Estratégia Saúde da Família na Atenção Primária à Saúde no Brasil: revisão da literatura. Ciências Saúde Coletiva. 2016; 21(5): 016; 1499-1510.

3. BARATIERI T, et al. Percepções de usuários atendidos em um Pronto Atendimento: olhar sobre a Atenção Primária à Saúde. 2017; 18(01).

4. BRASIL. Conselho Nacional de Secretários de Saúde. Atenção Primária Brasília: CONASS, 2011 a.

5. BRASIL. Ministério da Saúde. PORTARIA № 2.436, DE 21 DE SETEMBRO DE 2017. Brasília-DF, 2017 b.

6. BRAZ FAF. A importância do acolhimento aos usuários da atenção básica: uma assistência humanizada. Trabalho de Conclusão de Curso. Especialização em Atenção Básica em Saúde da Família. Universidade Federal de Minas Gerais. Campos Gerais/Minas Gerais. 2013.

7. BRUNHAUSER AL, et al. Avaliação de serviços de saúde bucal: um estudo comparativo. Revista da faculdade de odontologia Universidade de Passo Fundo. 2013; 18: 1.

8. CABRAL KFD, et al. Análise da Eficiência na Atenção Primária à Saúde sob a Ótica dos Profissionais da Área. Revista de Gestão em Sistemas de Saúde, 2016; 5(2): 71-83.

9. CONILL EM. Ensaio histórico-conceitual sobre a Atenção Primária à Saúde: desafios para a organização de serviços básicos e da Estratégia Saúde da Família em centros urbanos no Brasil. Cad Saude Publica. 2008; 24(Supl. 1): 7-27.

10. DEMARZO MMP. Módulo Político Gestor. Reorganização dos Sistemas de Saúde: Promoção da Saúde e Atenção Primária à Saúde. Especialização em Saúde da Família. Modalidade à distância. Universidade Federal de São Paulo. Universidade Aberta do SUS. 2011.

11. DIAS OV, et al. Acesso às consultas médicas nos serviços públicos de saúde. Revista Brasileira de Medicina de Família e Comunidade. 2016; 11: 38.

12. FAUSTO MCR, et al. A posição da Estratégia Saúde da Família na rede de atenção à saúde na perspectiva das equipes e usuários participantes do PMAQ-AB. Saúde Debate, 2014; 38( n.spe): 13-33.

13. GIOVANELA L, et al. Saúde da família: limites e possibilidades para uma abordagem integral de atenção primária à saúde no Brasil. CienSaude Colet; 2009; 14(3): 783-794.

14. GONTIJO TL, et al. Avaliação da atenção primária: o ponto de vista de usuários. Saúde Debate, 2017; 41(114): 741752.

15. GUIMARÃES FT, et al. Educação, saúde e ambiente: as concepções dos agentes comunitários de saúde. Ensino, Saúde e Ambiente, 2013; 6(1).

16. MARTINES WRV, CHAVES EC. Vulnerabilidade e sofrimento no trabalho do agente comunitário de saúde no Programa de Saúde da Família. Revista da Escola de Enfermagem da USP, 2007; 41(3): 426-433.

17. MENDES EV. A Construção Social da Atenção Primária À Saúde. Brasília: Conselho Nacional de Secretários de Saúde - CONASS, 2015.

18. MENICUCCI TMG. O Sistema Único de Saúde, 20 anos: balanço e perspectivas. CadSaude Publica; 2009; 25(7): 1620-1625.

19. OPAS. Qualidade da atenção primária no Programa Mais Médicos. A experiência dos médicos e usuários. Brasília, D.F.: Organização Pan-Americana da Saúde; 2018.

20. ROSA WDAG, LABATE RC. Programa Saúde da Família: a construção de um novo modelo de assistência. Revista Latino-Americana de Enfermagem, 2005; 13(6): 1027-1034.

21. SENNA CMM, SANTOS AM. Atenção Primária à Saúde Na Agenda Pública Brasileira: Dilemas Entre Focalização e Universalidade Revista de Políticas Públicas, 2017; 21(1): 403-424.

22. SILVA NETO JMA, et al. Atenção da equipe de saúde bucal no atendimento domiciliar: Revisão integrativaRevista Eletrônica Acervo Saúde, 2019; 37: e1958. 